\title{
Correction to: Efficacy of fulvestrant in the treatment of postmenopausal women with endocrine-resistant advanced breast cancer in routine clinical practice
}

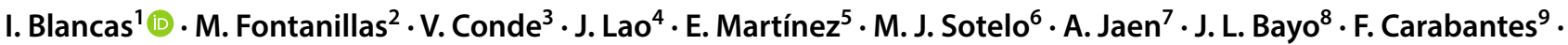

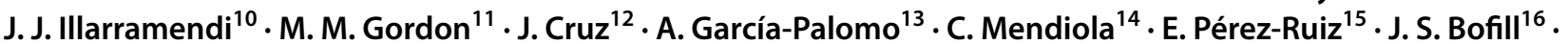 \\ J. M. Baena-Cañada ${ }^{17} \cdot$ N. M. Jáñez ${ }^{18} \cdot$ G. Esquerdo ${ }^{19} \cdot$ M. Ruiz-Borrego ${ }^{20}$
}

Published online: 3 October 2018

๑) Federación de Sociedades Españolas de Oncología (FESEO) 2018

\section{Correction to: Clin Transl Oncol (2018) 20:862-869 https://doi.org/10.1007/s12094-017-1797-9}

An information under Table 2 was published incorrectly. The correct data should read as follows:
Visceral involvement

No 22510.6 (8.9-11.6) 0.6604212121 (57.1) 0.7169 Yes $3810.0(7.1-13.9) 3619$ (52.8)

The corrected table is given in the following page:
The original article can be found online at https://doi.org/10.1007/ s12094-017-1797-9.

\section{Blancas}

iblancas@ugr.es

1 Hospital Universitario Clínico San Cecilio, Complejo Hospitalario Universitario, C/Dr. Oloriz, 16, 18012 Granada, Spain

2 Hospital Clinic de Barcelona, illarroel, 170, 08036 Barcelona, Spain

3 Hospital Universitario Virgen de las Nieves, Av. de las Fuerzas Armadas, S/N, 18014 Granada, Spain

4 Hospital Universitario Miguel Servet, Paseo Isabel la Católica, 1-3, 50009 Zaragoza, Spain

5 Hospital Provincial de Castellón, Av. del Dr. Clarà, 19, 12002 Castelló de la Plana, Spain

6 Hospital Universitario Clínico San Carlos, C/del Profesor Martín Lagos, s/n, 28040 Madrid, Spain

7 Hospital de Jaén, Avda. del Ejército Español, n 10, 23007 Jaén, Spain

8 Hospital Juan Ramón Jiménez, Ronda Exterior Norte s/n, 21005 Huelva, Spain

9 Hospital Universitario Carlos Haya, Av. de Carlos Haya, s/n, 29010 Málaga, Spain
10 Complejo Universitario Hospital de Navarra, Calle Irunlarrea, 3, 31008 Pamplona, Spain

11 Hospital de Jerez, Ronda de Circunvalación s/n, 11407 Cádiz, Spain

12 Hospital Universitario de Canarias, Carretera de Ofra, s/n, 38320 Santa Cruz de Tenerife, Spain

13 Complejo Universitario Asistencial de León, C/Altos de Nava, s/n, León, Spain

14 Hospital Universitario, 12 de Octubre, Avenida de Córdoba, s/n, 28041 Madrid, Spain

15 Hospital Costa del Sol, Autovia A-7, Km 187, 29603 Marbella, Spain

16 Hospital Nuestra Señora De Valme, Av. de Bellavista, s/n, 41014 Seville, Spain

17 Hospital Universitario Puerta del Mar, Av. Ana de Viya, 21, 11009 Cádiz, Spain

18 Hospital Ramón y Cajal, Ctra. de Colmenar Viejo, km. 9,100, 28034 Madrid, Spain

19 Clínica Benidorm, Av. Alfonso Puchades, 03501 Benidorm, Spain

20 Hospital Universitario Virgen del Rocío, Av. Manuel Siurot, s/n, 41013 Seville, Spain 
Table 2 Progression-free survival according to subgroups of patients

\begin{tabular}{|c|c|c|c|c|c|c|}
\hline & \multicolumn{3}{|c|}{ PFS (months) } & \multicolumn{3}{|c|}{$\mathrm{CBR}(\mathrm{CR}+\mathrm{PR}+\mathrm{SD} \geq 24$ months $)$} \\
\hline & $N$ & Median $(95 \% \mathrm{CI})$ & $p$ value & $N$ & $n(\%)$ & $p$ value \\
\hline \multicolumn{7}{|l|}{ Histologic type } \\
\hline Ductal & 208 & $11.2(9.9-13.0)$ & \multirow[t]{2}{*}{0.0002} & 196 & $115(58.7)$ & \multirow[t]{2}{*}{0.0635} \\
\hline Lobular & 37 & $7.8(5.6-10.9)$ & & 34 & $14(41.2 \%)$ & \\
\hline \multicolumn{7}{|l|}{ Hormonal receptors } \\
\hline $\mathrm{ER}+\mathrm{PgR}+$ & 197 & $10.8(9.1-12.1)$ & \multirow[t]{2}{*}{0.6725} & 189 & $114(60.3)$ & \multirow[t]{2}{*}{0.0088} \\
\hline $\mathrm{ER}+\mathrm{PgR}-$ & 61 & $8.9(6.8-11.2)$ & & 55 & $22(40.0 \%)$ & \\
\hline \multicolumn{7}{|l|}{ p53 } \\
\hline Positive $(>15 \%)$ & 19 & $13.6(5.8-\mathrm{NE})$ & \multirow[t]{2}{*}{0.4591} & 18 & $10(55.6)$ & \multirow[t]{2}{*}{1.0000} \\
\hline Negative & 95 & $9.2(7.6-10.9)$ & & 90 & $51(56.7 \%)$ & \\
\hline \multicolumn{7}{|l|}{ Ki67 } \\
\hline Positive $(>20 \%)$ & 70 & $9.6(6.5-13.1)$ & \multirow[t]{2}{*}{0.7224} & 66 & $34(51.5)$ & \multirow[t]{2}{*}{0.2917} \\
\hline Negative & 72 & $10.0(7.8-12.9)$ & & 66 & $41(62.1)$ & \\
\hline \multicolumn{7}{|l|}{ HER2 status } \\
\hline Positive & 26 & $10.2(7.7-13.3)$ & \multirow[t]{2}{*}{0.6809} & 24 & $12(50.0)$ & \multirow[t]{2}{*}{0.5209} \\
\hline Negative & 206 & $10.3(8.0-11.3)$ & & 196 & $112(57.1)$ & \\
\hline \multicolumn{7}{|l|}{ ECOG score } \\
\hline $0-1$ & 221 & $10.9(9.6-12.6)$ & \multirow[t]{2}{*}{0.0061} & 210 & $123(58.6)$ & \multirow[t]{2}{*}{0.3102} \\
\hline $2-3$ & 28 & $6.7(4.7-9.0)$ & & 27 & $13(48.1)$ & \\
\hline \multicolumn{7}{|l|}{ Visceral involvement } \\
\hline No & 225 & $10.6(8.9-11.6)$ & \multirow[t]{2}{*}{0.6604} & 212 & $121(57.1)$ & \multirow[t]{2}{*}{0.7169} \\
\hline Yes & 38 & $10.0(7.1-13.9)$ & & 36 & $19(52.8)$ & \\
\hline \multicolumn{7}{|l|}{ Treatment line } \\
\hline $1 \mathrm{st}$ & 59 & $11.5(9.5-19.8)$ & \multirow[t]{4}{*}{0.0245} & 56 & $31(55.4)$ & \multirow[t]{4}{*}{1.000} \\
\hline 2 nd & 84 & $10.6(8.3-13.6)$ & & 78 & $44(56.4)$ & \\
\hline $3 \mathrm{rd}$ & 52 & $9.9(6.7-13.3)$ & & 48 & $28(58.3)$ & \\
\hline$\geq 4$ th & 66 & $8.5(6.5-11.1)$ & & 64 & $35(54.7)$ & \\
\hline
\end{tabular}

$C B R$ clinical benefit rate, $C I$ confidence interval, $C R$ complete response, ECOG Eastern Cooperative Oncology Group, $E R$ estrogen receptor, $N E$ not evaluable, $P F S$ progression-free survival, $P R$ partial response, $P g R$ progesterone receptor, $S D$ stable disease 